\title{
Preparation and Characterization of Single-crystal Aluminum Nitride Substrates
}

\author{
Leo J. Schowalter, ,"** J. Carlos Rojo, ,* , Nikolai Yakolev", Yuriy Shusterman," \\ Katherine Dovidenko, ${ }^{*+}$ Rungjun Wang, Ishwara Bhat, ${ }^{*}$ and Glen A. Slack ${ }^{*, * *}$
}

\author{
*Rensselaer Polytechnic Institute \\ Troy, NY 12180 \\ *** Crystal IS, Inc. \\ Latham, NY 12110
}

\begin{abstract}
Large (up to $10 \mathrm{~mm}$ diameter) aluminum nitride (AlN) boules have been grown by the sublimation-recondensation method to study the preparation of high-quality single crystal substrates. The growth mechanism of the boules has been studied using AFM. It has been determined that large single crystal grains in those boules grow with a density of screw dislocations below $5 \times 10^{4} \mathrm{~cm}^{-3}$ while edge dislocations are at lower density (none were observed). High-quality AlN single crystal substrates for epitaxial growth have been prepared and characterized using Chemical Mechanical Polishing (CMP) and AFM imaging, respectively. Also, the differential etching effect of $\mathrm{KOH}$ solutions on the $\mathrm{N}$ and Al-terminated faces of AlN on vicinal c-faces has been investigated. In order to identify the $\mathrm{N}$ or Al-terminated face, convergent beam electron diffraction has been used.
\end{abstract}

\section{INTRODUCTION}

Single crystal AlN is attractive for the fabrication of substrates for III-nitride epitaxial growth. Applications of wide-bandgap and high-temperature semiconductors include the development of blue/UV solid-state charge injection lasers, UV optical sources and detectors, high power microwave devices, high power switches, and high temperature applications. Enhanced III-nitride epitaxy on AIN substrates, due to smaller lattice mismatch, smaller thermal expansion mismatch and good chemical compatibility, may allow substantial improvement in the ability to manufacture these devices. In addition, very low defect material is needed for developing solid-state low noise and power devices for use in power supplies, communications, fire control, surveillance, and multifunctional RF systems using the nitride, wide-bandgap semiconductors. Foreign substrate induced defects have also been implicated in the performance of high power microwave devices. Substrates of AlN are also attractive for surface acoustic wave (SAW) applications, which would take advantage of the very high sound velocity in AlN. This would have application in high power, high frequency amplifiers for wireless communication base stations. The high thermal conductivity and electrically insulating properties of AlN are also attractive for high performance substrates where heat sinking is a key issue.

\footnotetext{
${ }^{+}$Currently at the University at Albany, NY 12222
} 
For III-nitride epitaxial growth, the preparation of nearly-atomically flat surface is required. Due to the extreme hardness of AIN, we have found that epitaxial-growth quality surfaces can be only achieved by using chemical-mechanical polishing (CMP) methods. The determination of the etching properties of single crystal substrates is of great importance for epitaxial growth and device preparation. We have studied the effects of $\mathrm{KOH}$ solution on the both the $\mathrm{N}$ and Al-terminated surfaces along the c-axis crystallographic direction on high-quality single crystal substrates. Also, we discuss a TEM-based method to unambiguously identify the $\mathrm{N}$ and Al-terminated faces along the c-axis.

\section{CRYSTAL GROWTH OF BULK ALN}

Several (see Fig. 1) AlN boules of up to $10 \mathrm{~mm}$ of diameter have been produced at Crystal IS facilities using a sublimation-recondensation technique first developed for this material by Slack and McNelly [1], which demonstrated crystal growth rates up to $0.3 \mathrm{~mm} / \mathrm{hr}$. AFM imaging of the as-grown facets reveals interesting information about the growth mechanism of AlN. The atomic arrangement of the facets on the growing front of the crystal, shown in figure 2, corresponds to the characteristic structure of c-face fronts of hexagonal wurzite-type crystals. The $0.25 \mathrm{~nm}$-high monolayer steps consist of segments of triangles that form $60^{\circ}$ angles at their corners. The triangles on each successive monolayer are rotated $60^{\circ}$ or $180^{\circ}$ with respect to the preceding one. A step going in [ $1 \overline{1} 00$ ] direction has only 1 broken bond per edge atom in one layer. A parallel step in the next layer would have 2 broken bonds per edge atom; a step having again 1 broken bond per edge atom in the latter layer runs at $60^{\circ}$ angle with respect to the [1 $1 \overline{1} 00]$ step in the former layer. The straight segments of the steps indicate that the migration length of atoms along the steps is far more than a micron. The diffusion length of the atoms on the terraces must be even higher than that. We found that the origin of the step flow results from screw dislocations (see figure 3) which intersect the growing surface. The density of screw dislocations has been estimated to be $\sim 5 \cdot 10^{4} \mathrm{~cm}^{2}$. It is seen that the apex starts from the double step, hence the normal component of the Burgers vector is $\mathrm{c}=0.498 \mathrm{~nm}$. The step edge has the shape of the Archimede spiral without distinct straight segments. This means that the crystal growth is governed not only by the diffusion but also by the strain field of the dislocation, i.e., positions of an atom at the same distance of the dislocation are equally favorable without respect to the number of broken bonds.

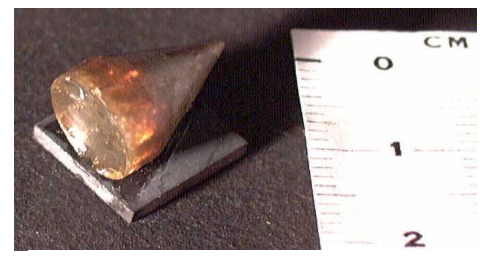

Figure1. Example of AlN

boule grown at Crystal IS. 


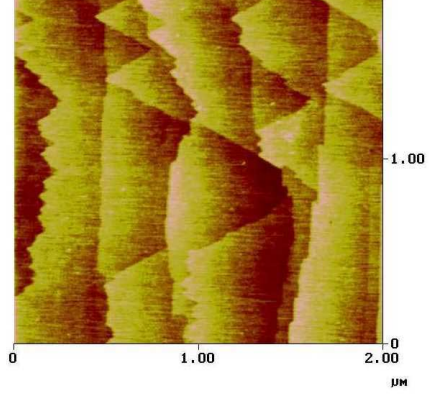

Figure 2. AFM image of the step flow growth mechanism of (0001)

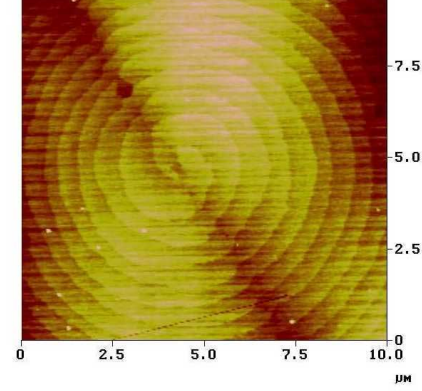

Figure 3. AFM image of a screw dislocation on a (0001) facet on the as-grown surface of AlN

\section{EPITAXIAL GROWTH ON A-FACE SUBSTRATES}

Boules of AlN have been cut to obtain several c-face and a-face substrates. Due to the extreme hardness of AlN, preparing high quality surfaces by common mechanical polishing process is both time-consuming and difficult. Mechanical polishing using diamond powder was not successful in obtaining an adequate surface for epitaxial growth as reported in a previous work [2]. Chemical-Mechanical Polishing (CMP) was, however, very successful in achieving epitaxial-growth-quality surfaces. Examination of the substrate by AFM showed a nearly atomically flat surface with monolayer steps and with all evidence of mechanical damage (sawing damage, scratches, etc.) removed. Rutherford backscattering/ion channeling (RBS) spectra were used to determine the crystal quality of the substrate surface and the grown epitaxial layers. The minimum yield $\chi_{\min }$ is used as a measure of the crystal quality and is defined as the ratio between the backscattered ion yield when the incident beam is aligned along a particular crystallographic axis and the backscattered ion yield when the incident beam is aligned randomly (along some noncrystallographic direction). The $\chi_{\text {min }}$ will increase with increasing defect density. We have measured $\chi_{\min }$ in the spectral window corresponding to ion scattering from immediately below the sample surface peak. The results (see figure 4) show a $\chi_{\text {min }}$ 's of $1.4 \%$ along the $[11 \overline{2} 0]$ axis of one of our AIN substrate after CMP. This value is very close to the theoretical minimum indicating that the CMP process is successful in removing mechanical damage near the surface of the substrate and was consistent with the AFM results. After growing a $0.7-\mu \mathrm{m}$-thick $\mathrm{AlN}$ and a $1-\mu \mathrm{m}$-thick $\mathrm{Al}_{0.5} \mathrm{Ga}_{0.5} \mathrm{~N}$ epitaxial layer, RBS spectra were also used to determine the crystal quality of the epitaxial layers. Details on the epitaxial growth process and the ion-channeling results can be found in Ref. (Schowalter Tucson). For the $0.7-\mu \mathrm{m}$-thick homoepitaxial layer of AlN, the $\chi_{\min }$ remained $1.5 \%$ again indicating excellent crystal quality. The $\chi_{\min }$ measured for the $\mathrm{Al}_{0.5} \mathrm{Ga}_{0.5} \mathrm{~N}$ layer was $2.2 \%$, which is still excellent considering that no attempt was made to optimize the growth parameters and that no buffer layer was needed to achieve them.

\section{DETERMINATION OF THE ETCHING EFFECTS ON VICINAL C-FACES}

Several studies performed on polycrystalline AlN have shown high etch rates obtain by using $\mathrm{KOH}$ based solutions. However, very little work has been done on etching of AlN 
single crystals. Recently, Mileham et al. [3] reported the first etching study on AlN single crystal grown on $\mathrm{Al}_{2} \mathrm{O}_{3}$. It was found that the etching rate depends critically on the crystalline quality and that the etching process is much faster at the grain boundary or wherever the density of defects is higher. Nevertheless, those crystal layers were defective and they do not report any orientation-dependent etching effect. The production of large, high-quality AlN single crystal substrates has made it possible to perform a more detailed study on the effect of $\mathrm{KOH}$-based solutions on this material and to distinguish the differences of the etching process on different crystal orientations.

During the preparation of a vicinal c-face substrate (cut $20^{\circ}$ off axis), we observed that cleaning the substrate with a phosphoric/sulfuric acid mixture gave different results for the two sides of the substrate. One side, which turned out to be the N-terminated face, became very rough surface. When a substrate with the same orientation was submerged in 1:2.5 KOH:water (by weight) solution for 3 minutes, AFM observation of the sample revealed the same unequal result for each side of the substrate. However, the $\mathrm{KOH}$ solution resulted in a slower etching rate than that produced by the phosphoric acid. AFM imaging (see figure 5) revealed a surface consisting of pyramids with a height between 0.5 and $1.5 \mu \mathrm{m}$ high. This surface cannot be considered adequate for epitaxial growth. However, on the side that turned out to be the Al-terminated face the etching rate was unobservable resulting in a flatter surface.

\section{STUDY OF THE SURFACE POLARITY BY TEM}

In order to identify the atomic termination of the side that resulted in a larger etching effect on the vicinal c-face of our substrate, a TEM-based method was used. Transmission electron microscopy (TEM) was carried out using JEOL 2010 and JEOL 200CX operated at $200 \mathrm{kV}$. We used a conventional TEM sample preparation technique consisting of mechanical polishing followed by $\mathrm{Ar}^{+}$ion milling of the specimen. The MacTempas $^{\odot}$ program was used for calculations of the diffracted beam intensities. Large, electron-transparent areas of the crystal were studied by TEM using different sets of two-beam, bright-field/dark-field conditions. The imaging was done close to [11 $\overline{2} 0$ ]

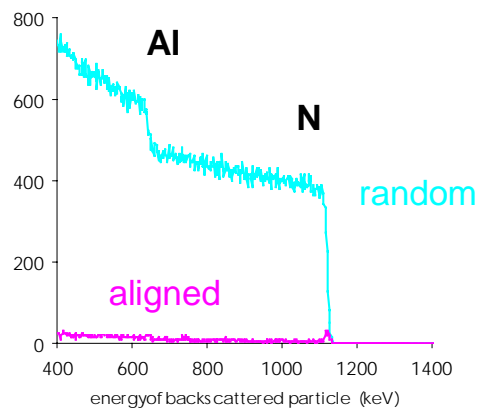

Figure 4. Ion-channeling results obtained on the a-face after CMP process

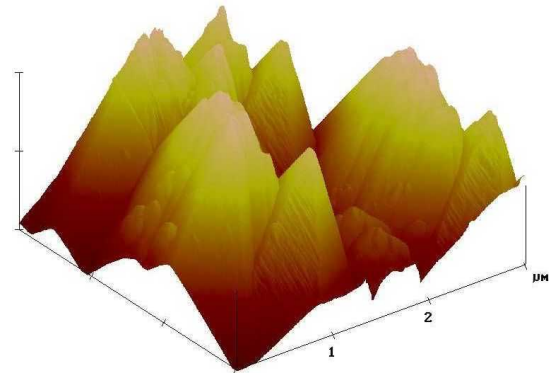

Figure 5. AFM image on a $2 \times 2 \mu \mathrm{m}^{2}$ area of the N-terminated c-face of AlN after $\mathrm{KOH}$ etching. Each height division represents $0.5 \mathrm{um}$ 
zone of AlN. No structural defects such as stacking faults or dislocations were observed over the studied area of about 80 square microns of the sample. A typical TEM image of the crystal taken under $g=10 \overline{1} 0$ bright field conditions is shown in figure 6 . Selected area electron diffraction patterns were taken from large (about 1.5 micron in diameter) and small (about 0.1 micron) areas of the AlN sample. No streaking of the diffraction spots or other indications of the possible defects in the sample was observed. An example of the [11 $\overline{2} 0$ ] AlN zone axis diffraction pattern taken from the area of 1.5 micron in diameter is shown in figure 7.

We have studied the polarity of the crystal surfaces using the method described by Dovidenko et al. [4]. The method is based on the specific dependence of diffracted beam intensity vs. specimen thickness for the diffracted beams revealing the noncentrosymmetric nature of the crystal structure. We have carried out calculation of the intensities of different diffracted beams for the [1120] zone of wurtzite AlN crystal structure using multi-slice image simulations. For our calculations, we assumed the positive [0001] direction of the structure to go from $\mathrm{Al}$ to $\mathrm{N}$ atom. The plots in figure 8 show the calculated intensities vs. crystal thickness for $0002,000 \overline{2}, 10 \overline{1} 2$ and $10 \overline{1} \overline{2}$ AlN diffracted beams. It is seen from the plot that, for the sample thickness in the range of $10 \mathrm{~nm}$, the intensity of the 0002 beam is $2-6$ times higher than the intensity of the $000 \overline{2}$ beam. This is due to the non-centrosymmetric nature of the crystal structure and the non-equivalency of the [0001] and [000 $\overline{1}]$ directions. Similar calculations were done for different diffracted beams belonging to the AlN [11 $\overline{2} 0]$ zone. To compare the intensities of the experimental diffracted beams and thus determine the polarity of the crystal, we obtained the convergent beam electron diffraction (CBED) patterns from the thin regions of the specimen. Since the intensity of the beams is a function of specimen thickness, we estimated the thickness by doing high-resolution TEM at the points where CBEDs were taken and comparing our high-resolution images with the calculated ones.

The experimental CBED pattern shown in figure 9 was obtained from a region of the

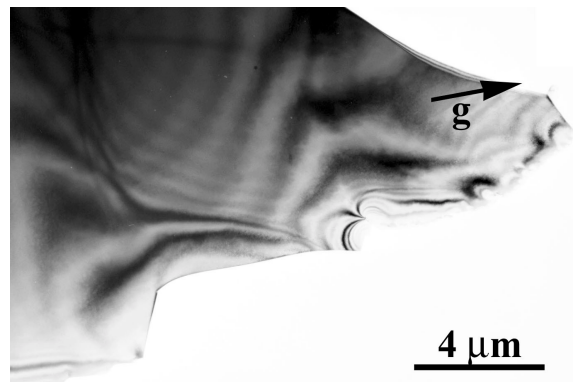

Figure 6. Typical TEM image of the crystal taken under $g=10 \overline{1} 0$ bright field conditions, the direction of $g$ vector is shown by arrow.

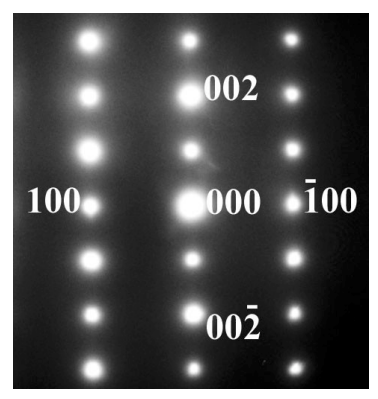

Figure 7. Selected area electron diffraction pattern of the AlN crystal taken from the area of about $1.5 \mu \mathrm{m}$ in diameter ([ $11 \overline{2} 0]$ zone axis). 


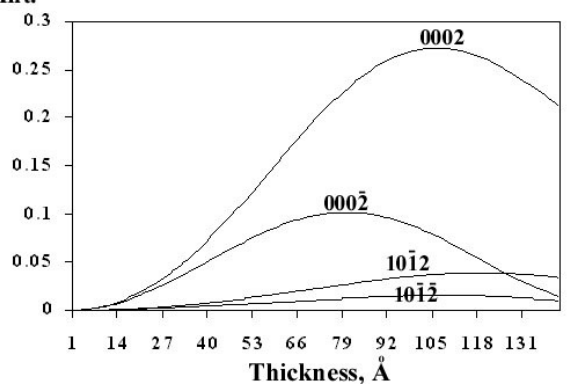

Figure 8. Calculated intensities (arbitrary units) of the 0002, $000 \overline{2}, 10 \overline{1} 2$ and $10 \overline{1} \overline{2}$ diffracted beams vs. sample thickness $(\AA)$ for I $11 \overline{2} 0$ l zone of wurtzite AlN crvstal structure.

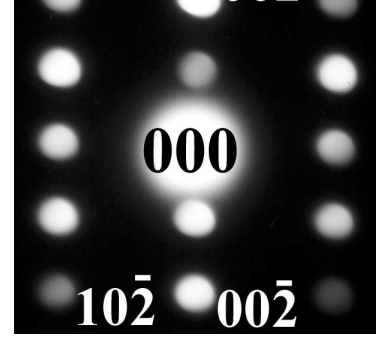

Figure 9. Convergent beam electron diffraction pattern taken from the thin (about $100 \AA$ ) region of the AlN crystal in $[11 \overline{2} 0]$ zone axis.

specimen which about $12 \mathrm{~nm}$ thick. The intensity of one of \{0002\}-type diffraction discs is significantly higher than that of the opposite one, the same is true for the $\{10 \overline{1} 2\}$-type of reflections. This allows the unambiguous indexing of the pattern and thus the determination of the absolute polarity of the crystal. The surface of the crystal, which was most affected by $\mathrm{KOH}$ etching, was found to be Al-terminated.

\section{CONCLUSIONS}

We have demonstrated the growth of large AIN boules with low-dislocation-density, single-crystal grains. High quality a-face AlN substrates have been prepared using a chemical-mechanical polishing technique. High quality epitaxial layers of AlN and $\mathrm{AlGaN}$ have been grown on substrates of this orientation without the need for buffer layers. Finally, we have reported the different morphology and behavior after $\mathrm{KOH}$ and phosphoric acid etching on the $\mathrm{N}$ and Al-terminated faces along the c-axis. The identification of these faces has been carried out with convergent beam electron diffraction.

ACKNOWLEDGEMENTS - Financial support from ONR, BMDO, and the US Navy is acknowledged as well as the support of our contract monitors Dr. C.E.C. Wood (ONR), Dr. C. Litton (AFRL), and T. Groshens (Naval Surface Warfare Center).

\section{REFERENCES}

1. G.A. Slack and T.F. McNelly, J. Crystal Growth 34, 263 (1976); and G.A. Slack and T.F. McNelly, J. Crystal Growth 42, 560 (1977).

2. J. Schowalter, J.C, Rojo, G.A. Slack, Y. Shusterman, R. Wang, I. Bhat, and G. Arunmozhi, J. Crystal Growth (accepted 99).

3. J.R. Mileham, S.J. Pearton, C.R. Abernathy, J.D. MacKenzie, R. J. Shul, S.P. Kilcoyne. Appl. Phys. Lett. 67, 1119 (1995).

4. K. Dovidenko, S. Oktyabrsky, J. Narayan, and M. Razeghi, MRS Internet J. Nitride Semicond. Res. 4S1, G6.46 (1999). 